\title{
The control algorithm of whole angle mode for HRG based on the vector composition
}

\author{
Wanliang Zhao ${ }^{1,2}$, Yuxiang Cheng ${ }^{1,2}$, Shaoliang Li $i^{1,2}$ and Lijun Song ${ }^{3 *}$
}

\section{*Correspondence:}

songlijun9071@sina.com

${ }^{3}$ School of Information

and Control Engineering,

Xi'an University

of Architecture

and Technology,

Xi'an 710055, Shaanxi, China

Full list of author information is available at the end of the article

\begin{abstract}
The Hemispherical Resonator Gyroscope (HRG) has many advantages such as high precision, high reliability and long life-time, it is widely used in the space-launcher and the satellites. The HRG has been mechanized to operate in the distinct operating modes, the Force to Rebalanced (FTR) mode and the Whole Angle (WA) mode. In the paper, different from the traditional control algorithm is based on average methods which usually used for the WA mode, a new electrostatic control algorithm is presented, which based on the vector composition and decomposition method to control the equivalent drive force in order to track the phase of the standing wave. The mathematics model and the control algorithm are presented in the paper, and the hardware experimental circuit system is implemented, the HRG has a range of more than $300 \%$ with the linearity of $40 \mathrm{ppm}$. Meanwhile, the energy transition efficiency increases by an order of magnitude.
\end{abstract}

Keywords: Whole angle mode (WA), Hemispherical resonator gyroscope (HRG), Vector composition, Energy transition efficiency, Phase tracking

\section{Introduction}

The HRG is an important kind of the inertial sensor based on the Coriolis' Effect. Due to the simple structure and fixed mechanical components, the HRG has the characteristics of small size, high reliability and long life-time [1]. The HRG has been mechanized to operate in two operating modes, the FTR mode and the WA mode. Compared with the FTR mode, the WA mode has high dynamic range, fast response and none of the rate integrated error.

In the case of the WA mode, the standing wave of the resonator is free to rotation around the body of the shell. The excitation axes are fixed by the driving electrodes and the fixed voltage is placed on the driving electrodes to maintain the fixed vibration energy of the resonator. However, due to the manufacture error of the resonator, besides of the angle precession according to the Coriolis' Effect, the standing wave is shift around the hemispheric shell, which will arouse the performance reduction.

The traditional control algorithm is based on average methods [2-5] which usually used for the WA mode. In the paper, a different method to sustain the vibration energy is presented and implemented. The different computed $\mathrm{AC}$ voltages are applied to the different

(c) The Author(s) 2021. Open Access This article is licensed under a Creative Commons Attribution 4.0 International License, which permits use, sharing, adaptation, distribution and reproduction in any medium or format, as long as you give appropriate credit to the original author(s) and the source, provide a link to the Creative Commons licence, and indicate if changes were made. The images or other third party material in this article are included in the article's Creative Commons licence, unless indicated otherwise in a credit line to the material. If material is not included in the article's Creative Commons licence and your intended use is not permitted by statutory regulation or exceeds the permitted use, you will need to obtain permission directly from the copyright holder. To view a copy of this licence, visit http:// creativecommons.org/licenses/by/4.0/. 
driving electrodes, which causing the standing wave to rotate, and a new electrostatic control algorithm is presented, which based on the vector composition and decomposition method to control the equivalent drive force in order to track the phase of the standing wave [6-8]. The efficiency of the energy transmission [9] is increased, and the sensitivity of the gyroscope is enhanced too.

\section{The vector composition}

In the active power filter topology which based on the voltage-source inverter, the switching frequency fluctuation of inverter can be reduced by the voltage space vector pulsewidth modulation method.. Compared with the sinusoidal pulse-width modulation, it has higher DC-side voltage utilization and can output better waveforms at lower switching frequencies, and also has more controllable switching devices for better control performance.

According to the requirement of separate and independent control for the HRG, the paper separates the zero-sequence and the non-zero-sequence components of the voltage by using an appropriate coordinate system. The definition of the space vector is followed in the $\alpha-\beta$ plane, which does not include the zero-sequence component; the 0 -axis is used as the third axis perpendicular to the $\alpha \beta$ axis, as shown in Fig. 1.

The three-phase imaginary axis coordinate system adopted as plane coordinate system, that is, the three axes of $\mathrm{a}, \mathrm{b}$ and $\mathrm{c}$ are rotated $90^{\circ}$ counterclockwise, then $X, Y, Z$ coordinates are obtained, as shown in Fig. 2.

The conversion formula of this coordinate system with the abc coordinate system and the $\alpha-\beta$ coordinate system is shown in formula (1).

$$
\left[\begin{array}{l}
x_{j_{a}} \\
y_{j_{b}} \\
z_{j c}
\end{array}\right]=\left[\begin{array}{cc}
0 & \sqrt{2} \\
-\sqrt{3 / 2} & -1 / \sqrt{2} \\
\sqrt{3 / 2} & -1 / \sqrt{2}
\end{array}\right]\left[\begin{array}{l}
x_{\alpha} \\
y_{\beta}
\end{array}\right],\left[\begin{array}{l}
x_{j_{a}} \\
y_{j_{b}} \\
z_{j c} \\
x_{0}
\end{array}\right]=\left[\begin{array}{ccc}
0 & 1 & -1 \\
-1 & 0 & 1 \\
1 & -1 & 0 \\
1 / 3 & 1 / 3 & 1 / 3
\end{array}\right]\left[\begin{array}{l}
x_{a} \\
y_{b} \\
z_{c}
\end{array}\right]
$$

This algorithm is very simple for the non-zero sequence component and action time of the composite vector in the imaginary axis coordinate system. It does not need

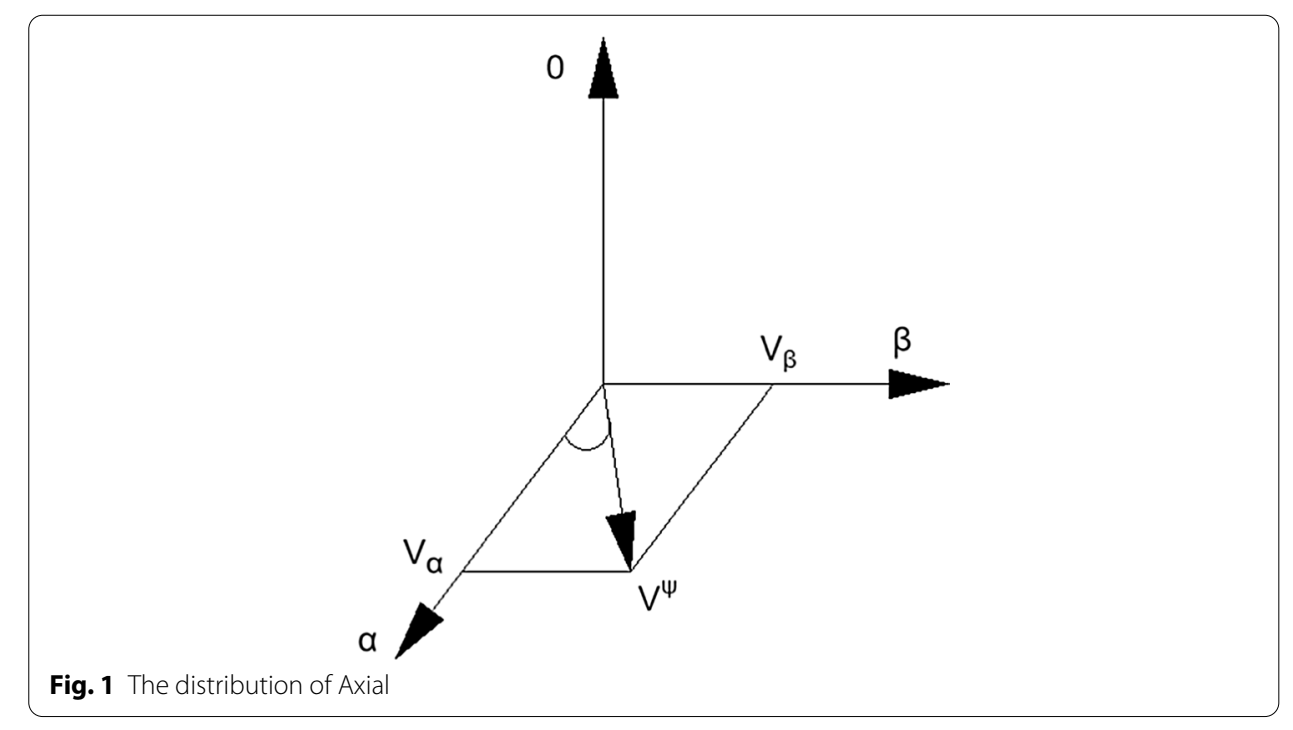




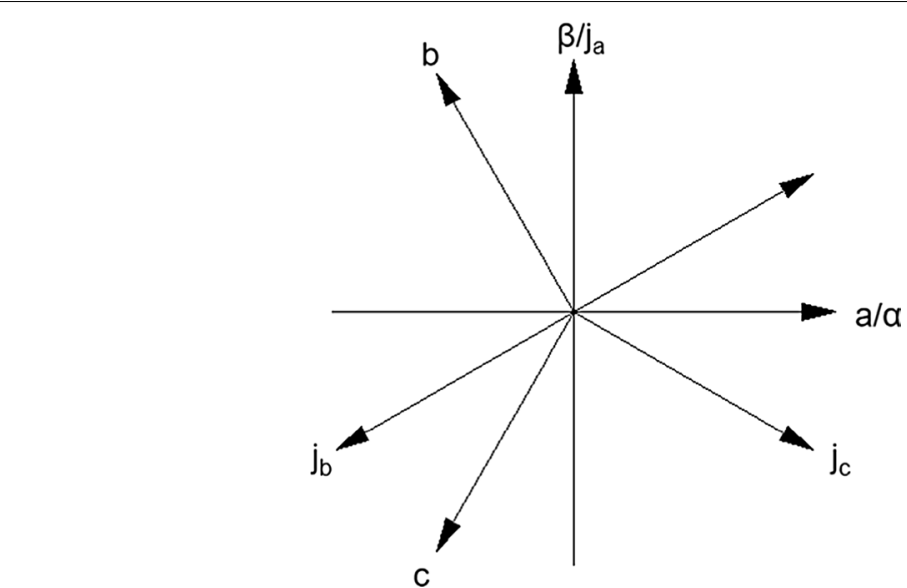

Fig. 2 The coordinate system of three phase virtual axis

multiplication, and there are very few addition and subtraction. The calculation is completely universal for any different electrical level. Moreover, according to different circuit forms and system requirements, this algorithm can design and implement flexible control of the zero-sequence voltage. The method of design and implementation is simple and can play the redundancy of the switching state of the multi-level inverter. So, it can be applied to a variety of circuit topologies, and really put it into practical use.

\section{The modelling of HRG}

In order to design the control and signal processing algorithms for the WA mode of HRG, it is necessary to establish the mathematical model of gyroscope output signal. And the mathematical model of gyroscope output signal can be obtained by solving the dynamic model of HRG resonator's vibrating.

The major component of the HRG is the hemispherical resonator, which will form a 2-order standing wave, and be excited by the external signal with the natural frequency of the resonator. Regarded the hemispherical resonator as a proof spring-damping model, the dynamics of the HRG resonator's vibrating can be simplified as follows [2, 10-12]:

$$
\begin{gathered}
\ddot{x}+\frac{2}{\tau} \dot{x}+\Delta\left(\frac{1}{\tau}\right)(\dot{x} \cos 2 \theta+\dot{y} \sin 2 \theta)+\left(\omega^{2}-k^{2} \Omega^{2}\right) x \\
-\omega \Delta \omega(x \cos 2 \theta+y \sin 2 \theta)-k(2 \Omega \dot{y}+\dot{\Omega} y)=0 \\
\ddot{y}+\frac{2}{\tau} \dot{y}-\Delta\left(\frac{1}{\tau}\right)(-\dot{x} \sin 2 \theta+\dot{y} \cos 2 \theta)+\left(\omega^{2}-k^{2} \Omega^{2}\right) y \\
+\omega \Delta \omega(-x \sin 2 \theta+y \cos 2 \theta)+k(2 \Omega \dot{x}+\dot{\Omega} x)=0
\end{gathered}
$$

In Eq. (1) of the dynamic model, where $x$ and $y$ are the vibrating motion in the quadrature axes; $\omega$ is the vibrating frequency; $\tau$ is the damping parameter; $\Delta \omega$ and $\Delta(1 / \tau)$ are the frequency splitting and the damping mismatch respectively; $\theta$ is the orientation of precession; $k$ is the gain factor of the gyroscope. The solution of Eq. (2) is 


$$
\left\{\begin{array}{l}
x=a \cos (\omega t) \cos \left(\phi_{0}\right)-q \sin (\omega t) \sin \left(\phi_{0}\right) \\
y=a \cos (\omega t) \sin \left(\phi_{0}\right)+q \sin (\omega t) \cos \left(\phi_{0}\right)
\end{array}\right.
$$

Equation (2) is the mathematical model of the gyroscope output signal and it can be regard as signal of the quadrature axes. In Eq. (3), $a$ is the amplitude of the vibration, $q$ is the amplitude of quadrature, $\phi_{0}$ is the precession angle. Using the averaging derivative of precession angle is [3]:

$$
\dot{\phi}_{0}=-k \Omega+\frac{1}{2} \Delta\left(\frac{1}{\tau}\right) \sin (2 \theta)+\Delta \omega \cos (2 \theta) \frac{a q}{a^{2}-q^{2}}
$$

Equation (3) shows that anis-elasticity and anis-damping will lead to a drift of the vibrating pattern and add an error item into the detected angle signal.

\section{The control algorithm of the WA mode}

The control algorithm of the WA mode is including the energy control loop, the quadrature control loop, the frequency tracking loop, the real-time angle calculation and the electrostatic control forcer. The control scheme is shown in Fig. 3.

The energy is supplied by the energy control loop and keeps the standing wave's amplitude stable. The precision of the gyroscope is improved by the null quadrature error of the quadrature control loop. The real-time frequency of resonator can be tracked by the frequency tracking loop and provided reference frequency to calculation procession. The precession angle can be calculated by the angle calculation. The control force can be transferred to driving vector by the electrostatic control force driver and applied to the resonator.

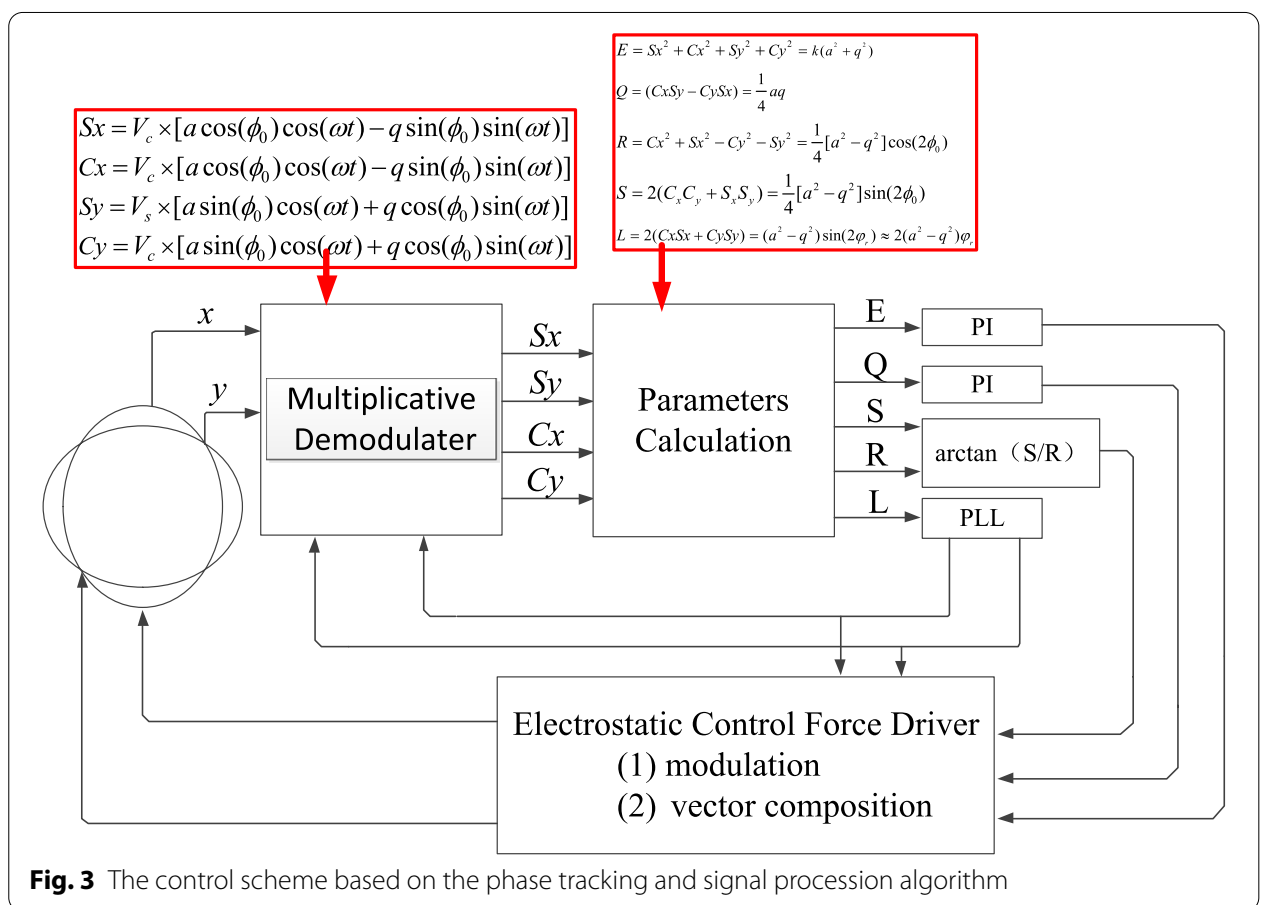




\subsection{The feedback parameter}

E, Q, S, R, L are feedback information for the WA mode control loop of HRG, and $t$ implicit in the output signal of $\mathrm{x}$ and $\mathrm{y}$ of the gyroscope. To extract the information, the multiplicative demodulator is needed to take low frequency signal which contain the high frequency noise signal. The calculation process of the multiplicative demodulator is showed as Eq. (5), where $V s, V c$ are the output signal of the frequency tracking loop, and $S x, C x, S y, C y$ are the output of the multiplicative demodulator.

$$
\begin{aligned}
S x & =V_{c} \times\left[a \cos \left(\phi_{0}\right) \cos (\omega t)-q \sin \left(\phi_{0}\right) \sin (\omega t)\right] \\
C x & =V_{c} \times\left[a \cos \left(\phi_{0}\right) \cos (\omega t)-q \sin \left(\phi_{0}\right) \sin (\omega t)\right] \\
S y & =V_{s} \times\left[a \sin \left(\phi_{0}\right) \cos (\omega t)+q \cos \left(\phi_{0}\right) \sin (\omega t)\right] \\
C y & =V_{c} \times\left[a \sin \left(\phi_{0}\right) \cos (\omega t)+q \cos \left(\phi_{0}\right) \sin (\omega t)\right] \\
V_{s} & =\sin \left(\omega t+\varphi_{r}\right) \\
V_{c} & =\cos \left(\omega t+\varphi_{r}\right)
\end{aligned}
$$

Then, according to Eq. (6), E, Q, S, R, L can be calculated from the low frequency signal.

$$
\begin{aligned}
& E=S x^{2}+C x^{2}+S y^{2}+C y^{2}=k\left(a^{2}+q^{2}\right) \\
& Q=(C x S y-C y S x)=\frac{1}{4} a q \\
& R=C x^{2}+S x^{2}-C y^{2}-S y^{2}=\frac{1}{4}\left[a^{2}-q^{2}\right] \cos \left(2 \phi_{0}\right) \\
& S=2\left(C_{x} C_{y}+S_{x} S_{y}\right)=\frac{1}{4}\left[a^{2}-q^{2}\right] \sin \left(2 \phi_{0}\right) \\
& L=2(C x S x+C y S y)=\left(a^{2}-q^{2}\right) \sin \left(2 \varphi_{r}\right) \approx 2\left(a^{2}-q^{2}\right) \varphi_{r}
\end{aligned}
$$

\subsection{The energy control loop}

The energy control loop is designed to maintain the vibration amplitude of the resonator. It is compared the feedback parameter E with the set value of the energy and used the PI controller to adjust the control force of the energy loop. In the PI controller, the P parameter effect the response time of the energy control loop, the I parameter effect the accuracy of the steady state and the set-value is determined by the electrode spacing parameter of the HRG.

\subsection{The quadrature control loop}

Because of the additional errors which caused by the quadrature error shown as Eq. (3), the quadrature error will arise the vibrating pattern's drift. The quadrature control loop is designed to the null quadrature error and maintain the wave node amplitude at 0 . As the energy control loop, the PI controller is also need here.

\subsection{The frequency tracking loop}

In Eq. (6), L can be regarded as the frequency difference between the real-time frequency of the resonator and the output signal of the frequency tracking loop. By adjusting the frequency of $\mathrm{VCO}$ in the frequency tracking loop, until $\mathrm{L}$ is 0 , 
the frequency of the output signal will be equal to the real-time frequency of the resonator.

\subsection{The angle calculation}

The angle is calculated out according to Eq. (7). The information of processing angle and the amplitude of vibration are contained in the parameter $\mathrm{S}$ and $\mathrm{R}$. The information of amplitude is eliminated by division elimination of $S$ and $R$.

$$
\phi_{c}=\arctan \left(\frac{S}{R}\right)=\arctan \left(\frac{\frac{1}{4}\left[a^{2}-q^{2}\right] \sin \left(2 \phi_{0}\right)}{\frac{1}{4}\left[a^{2}-q^{2}\right] \cos \left(2 \phi_{0}\right)}\right)=\arctan \left(\frac{\sin \left(2 \phi_{0}\right)}{\cos \left(2 \phi_{0}\right)}\right)=2 \phi_{0}
$$

\subsection{The control force applying}

Because of the vibrating pattern's free procession, it's difficult to apply the control force to the resonator directly, just as the WA mode based the average methods. So, it can apply the electrostatic control forcer based on the phase tracking method to solve the problem.

There are 16 driving electrodes in the electrostatic control force driver. The electrodes are evenly distributed around the resonator, and divided into 4 groups. The control force which is formed the energy control loop and FE\& FQ modulated of the quadrature control loop are applied to the resonator by 4 groups of the electrodes, which are according to the vector composition and the decomposition method as the Eqs. (8)-(11).

The electrodes at position of $0^{\circ}, 90^{\circ}, 180^{\circ}, 270^{\circ}$ are included in the first group of the electrodes. The control force is from these electrodes as $F_{0}, F_{90}, F_{180}, F_{270}$ in Eq. (8).

$$
\left\{\begin{array}{l}
F_{0}=F_{a} \cos (\omega t) \cos \left(\phi_{0}\right) \\
F_{90}=-F_{a} \cos (\omega t) \cos \left(\phi_{0}\right) \\
F_{180}=F_{a} \cos (\omega t) \cos \left(\phi_{0}\right) \\
F_{270}=-F_{a} \cos (\omega t) \cos \left(\phi_{0}\right)
\end{array}\right.
$$

The electrodes at position of $45^{\circ}, 135^{\circ}, 225^{\circ}, 315^{\circ}$ are included in the second group of the electrodes. The control force is from these electrodes as $F_{45}, F_{135}, F_{225}, F_{315}$ in Eq. (9).

$$
\left\{\begin{array}{l}
F_{45}=F_{a} \cos (\omega t) \sin \left(\phi_{0}\right) \\
F_{135}=-F_{a} \cos (\omega t) \sin \left(\phi_{0}\right) \\
F_{225}=F_{a} \cos (\omega t) \sin \left(\phi_{0}\right) \\
F_{315}=-F_{a} \cos (\omega t) \sin \left(\phi_{0}\right)
\end{array}\right.
$$

The electrodes at position $22.5^{\circ}, 112.5^{\circ}, 202.5^{\circ}, 292.5^{\circ}$ are included in the third group of the electrodes. The control force is from these electrodes as $F_{22.5}, F_{112.5}, F_{202.5}, F_{292.5}$ in Eq. (10).

$$
\left\{\begin{array}{l}
F_{22.5}=F_{q} \\
F_{112.5}=F_{q} \\
F_{202.5}=F_{q} \\
F_{292.5}=F_{q}
\end{array}\right.
$$


The electrodes at position of $67.5^{\circ}, 157.5^{\circ}, 247.5^{\circ}, 337.5^{\circ}$ are included in the fourth group of the electrodes. The control force is from these electrodes as $F_{67.5}, F_{157.5}, F_{247.5}$ , $F_{337.5}$ in Eq. (11).

$$
\left\{\begin{array}{l}
F_{67.5}=-F_{q} \\
F_{157.5}=-F_{q} \\
F_{247.5}=-F_{q} \\
F_{337.5}=-F_{q}
\end{array}\right.
$$

\section{The experimental of HRG}

In the part III,the hardware experimental circuit based on the WA mode of HRG are consists of FPGA and electro-mechanical interface. The energy control loop, the quadrature control loop, the frequency tracking loop and the real-time angle calculation part are all realized in FPGA. The hardware experimental platform and the HRG are shown as Fig. 4.

\subsection{The rotation rate test}

The dynamic range of rotation rate of the HRG [13-15] and the linearity of the output angle are tested in the experiment.

In order to test the dynamic performance of the HRG at the different angular velocities, the output data of the HRG are recorded under the rotation of $\pm 50^{\circ} / \mathrm{s}, \pm 100^{\circ} / \mathrm{s}$, $\pm 300^{\circ} / \mathrm{s}$ and plotted the graphs as Fig. 5. The slope rate of curves in the Fig. 3 represents the output rotation rate. The gain factor of the HRG at different rotation speed is approximate, about 0.2757 . And the linearity of the gain factor is better than $40 \mathrm{ppm}$ without any signal compensation.

\subsection{The energy control loop test}

To compare with the phase tracking and the average method, two energy control methods are implemented with the same HRG and the same hardware circuit. The

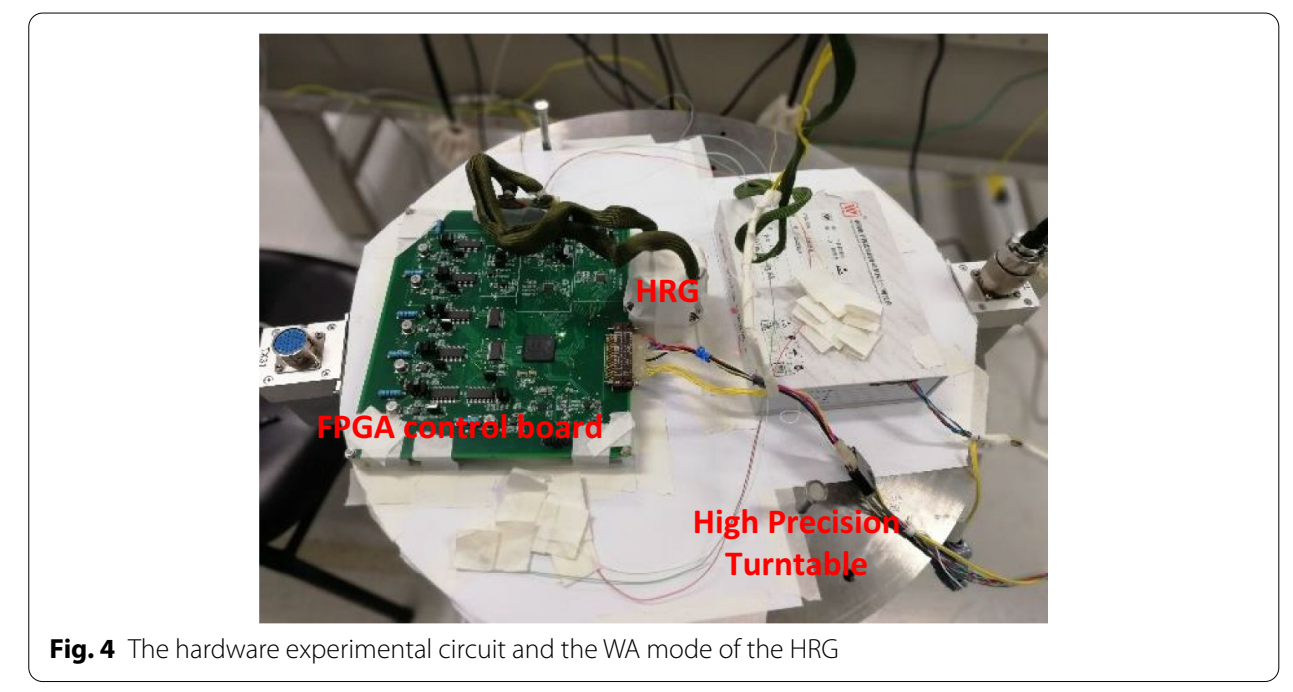



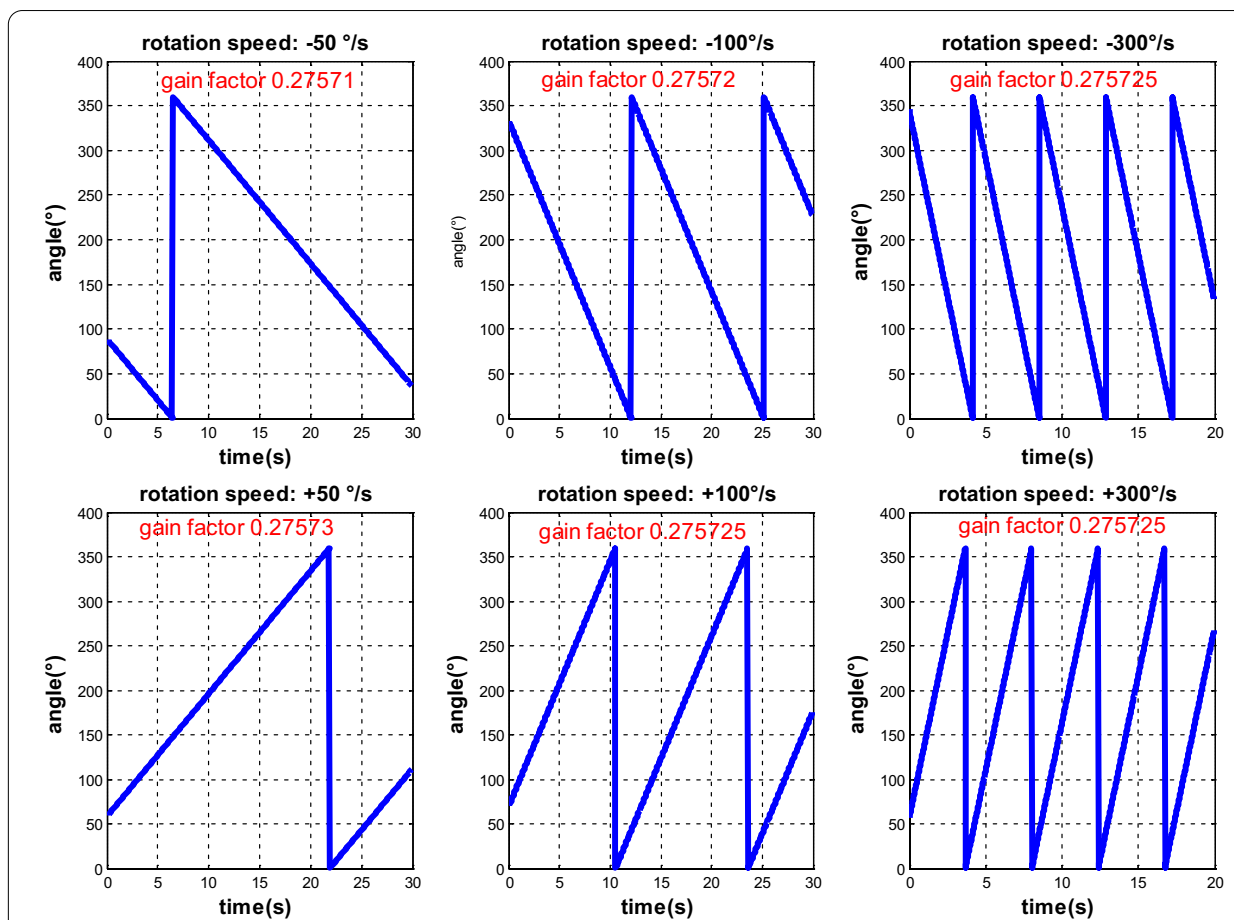

Fig. 5 The output data of the HRG at different rotation rate

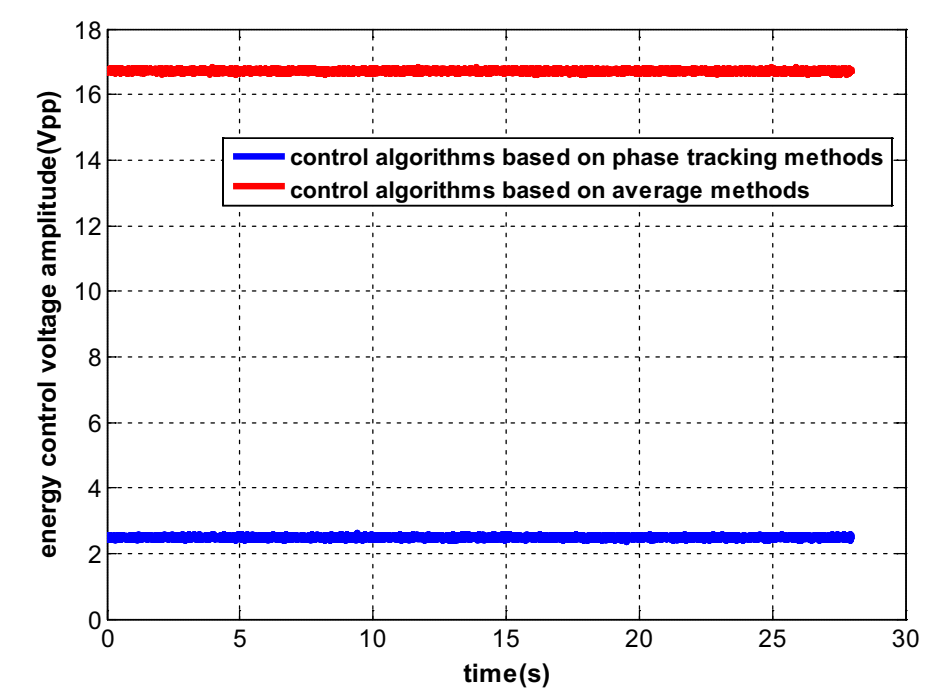

Fig. 6 The energy control loop voltage in two methods

energy control voltages are recorded by upper-computer software, as shown in Fig. 6. Obviously, the phase tracking method in the paper, the energy control voltages could decrease an order of magnitude. It means that the energy transition efficiency increases by an order of magnitude. 


\section{The result and summary}

In the paper, in order to improve the energy transition efficiency of the HRG and the null accumulative error of the angle detect, the control scheme of the HRG's WA mode with the energy control loop by phase tracking methods, the control scheme include the energy control loop, the quadrature control loop, the frequency tracking loop, the realtime angle calculation and the electrostatic control forcer and the mathematics models are derivate in the paper. The results of the experiments show that the dynamic range of the rate detection is better than $360 \%$ s and the linearity of the angle detection is better than $40 \mathrm{ppm}$. The energy transition efficiency with phase tracking method could increase by an order of magnitude. In the future works, the further electrostatic balance and signal compensation, the performance of the control scheme will have been improved greatly.

\section{Abbreviations}

HRG: Hemispherical Resonator Gyroscope; FTR: Force to Rebalanced; WA: Whole Angle.

\section{Authors' contribution}

WL participated in the sequence alignment and drafted the manuscript. YX and SL participated in the design of the study and performed the statistical analysis. $L J$ conceived of the study, and participated in its design and coordination and helped to draft the manuscript. All authors read and approved the final manuscript.

\section{Funding}

This work is partially sponsored by the National Key Research and Development Program of China under the Grant no.2017YFB1 104700, the Natural Science Foundation of Shaanxi under Project 2020JM-488 and Shanghai Rising Star Program under 20QA1404300.

\section{Data availability statement}

The raw/processed data required to reproduce these findings cannot be shared at this time as the data also forms part of an ongoing study.

\section{Competing interest}

We declare that we do not have any commercial or associative interest that represents a conflict of interest in connection with the work submitted.

\section{Author details}

1 Shanghai Aerospace Control Technology Institute, Shanghai 201109 , China. ${ }^{2}$ Shanghai Engineer Research Center of Inertia, Shanghai 201109, China. ${ }^{3}$ School of Information and Control Engineering, Xi'an University of Architecture and Technology, Xi'an 710055, Shaanxi, China.

Received: 22 November 2020 Accepted: 28 January 2021

Published online: 15 February 2021

\section{References}

1. P. Shao, C.L. Mayberry, X. Gao, V. Tavassoli, F. Ayazi, A Polysilicon micro hemispherical resonating gyroscope. J. Microelectromech. Syst. 23(4), 762-764 (2014)

2. D.D. Lynch, Vibratory Gyro Analysis by the Method of Averaging, Proceedings 2nd Saint Petersburg Int. Conf. on Gyroscopic Technology and Navigation, pp. 26-34, 1995.

3. M. Song, et al. Control scheme and error suppression method for micro rate integrating gyroscopes. Guidance, Navigation \& Control Conference IEEE, 2017.

4. P. Taheri-Tehrani, et al. "Disk resonator gyroscope with whole-angle mode operation." IEEE International Symposium on Inertial Sensors \& Systems IEEE, 2015.

5. J.-K. Woo, J.Y. Cho, C. Boyd, and K Najafi, Whole-angle-mode micro-machined fused-silica birdbath resonator gyroscope (WA-BRG), MEMS 2014, San Francisco, CA, USA, January 26 - 30, 2014

6. D.D. Lynch, R.R. Savava, J.J. Campanile, Hemispherical Resonator Gyro Control. USA: 7,318,347 B2, Jan. 15, 2008

7. P. Taheri-Tehrani, O. Izyumin, I. Izyumin, C.H. Ahn, E.J. Ng, V.A. Hong, Y. Yang, T.W. Kenny, B.E. Boser, D.A. Horsley. Disk resonator gyroscope with whole-angle mode operation. IEEE International Symposium on Inertial Sensor \& System.2015:1-4

8. V. Apostolyuk. Whole Angle Force Rebalance Control for Coriolis Vibratory Gyroscopes. 2014 IEEE 3rd International Conference on Methods and Systems of Navigation and Motion Control(MSNMC) Proceedings: pp.59-61

9. M. Zhou, Y. Wang, Z. Tian, Y. Lian, Y. Wang, B. Wang, Calibrated data simplification for energy-efficient location sensing in internet of things. IEEE Internet Things J. 6(4), 6125-6133 (2019) 
10. H.H. Ge, J.Y. Liu, Bryan Buchanan, Bias Self-calibration Techniques using Silicon Disc Resonator Gyroscope,The Boeing Company

11. J.-K. Woo, J.Y. Cho, C. Boyd, K. Najafi. Whole Angle Mode Micro-Machined Fused-Silica Birdbath Resonator Gyroscope. MEMS 2014, San Francisco, CA, USA, January 26-30, 2014

12. A.A. Trusov, G. Atikyan, D.M. Rozelle, A.D. Meyer S. A. Zotov, B.R. Simon, A.M. Shkel. Force Rebalance, Whole Angle, and Self-Calibration Mechanization of Silicon MEMS Quad Mass Gyro. International Symposium on Inertial Sensor \& System.2014:1-2

13. A.A. Trusov, I.P. Prikhodko, S.A. Zotov, A.M. Shkel, Low-dissipation silicon tuning fork gyroscopes for rate and whole angle measurements. IEEE Sens. J. 11, 2763-2770 (2011)

14. L. Song, Z. Duan, B. He, Z. Li. Application of Federal Kalman Filter with neural networks in the velocity and attitude matching of Transfer Alignment. Complexity, Volume 2018 (2018), Article ID 3039061, 7 pages

15. D.D. Lynch. CVGs Utilizing Non-Axisymmetric Structures Operating in Whole-Angle Mode. IEEE International Symposium on Inertial Sensor \& System.2014:1-4

\section{Publisher's Note}

Springer Nature remains neutral with regard to jurisdictional claims in published maps and institutional affiliations.

\section{Submit your manuscript to a SpringerOpen ${ }^{\circ}$} journal and benefit from:

- Convenient online submission

- Rigorous peer review

Open access: articles freely available online

- High visibility within the field

Retaining the copyright to your article

Submit your next manuscript at $\gg$ springeropen.com 